\title{
On the Storage Capacity of nonlinear Neural Networks
}

\author{
Christian Mazza* \\ Department of Mathematics \\ University of Geneva
}

December 20, 1994

\begin{abstract}
We consider artificial neural networks (ANN) of the Hopfield type. Under some assumptions on the nonlinear synaptic function $G$ we give a rigorous lower bound for the storage capacity of the (ANN). That is, given an (ANN) with $n \in \mathbb{N}$ neurons and $m=m(n) \in \mathbb{N}$ patterns to be stored, we show that retrieval without errors occurs when $m(n) \rightarrow \infty$ as $n \rightarrow \infty$ in such a way that $m(n)<(n / 2 \log (n)) q_{G}$, where $0<q_{G}:=$ $\left(E(N G(N))^{2} / E\left(G(N)^{2}\right)\right) \leq 1$, and $N$ denotes a standard normal random variable.
\end{abstract}

*This research was supported in part by the Swiss National Science Foundation and by the Institute for Mathematics and its Applications with funds provided by the US National Science Foundation 


\section{Introduction}

We investigate the capacity of nonlinear artificial neural networks ( $\mathrm{NNN}$ ) in the discrete time discrete state space setting. At any time, the dynamical system is characterized by a binary vector

$$
X(t)=\left(x_{1}(t), . ., x_{n}(t)\right)^{t r}
$$

where $x_{i}(t) \in\{-1,+1\}, 1 \leq i \leq n, \forall t \in \mathbb{N}$. Given a real symmetric synaptic matrix $W=\left(w_{i j} ; 1 \leq i \neq j \leq n\right)$, and a threshold vector $\theta=\left(\theta_{1}, . ., \theta_{n}\right)^{t r}$, the time evolution of the dynamical system is given by (see e.g. [1])

$$
x_{i}(t+1) \equiv \operatorname{Sgn}\left(\sum_{j=1}^{n} w_{i j} x_{j}(t)-\theta_{j}\right),
$$

with the convention that

$$
\sum_{j=1}^{n} w_{i j} x_{j}(t)=\theta_{j} \Rightarrow x_{j}(t+1)=x_{j}(t)
$$

Let us describe the storage problem. Given a set of $m \in \mathbb{N}$ patterns $\xi^{(1)}, \ldots, \xi^{(m)}$, where $\xi^{(i)} \in\{-1,+1\}^{n}, 1 \leq i \leq m$, find a synaptic matrix $W$ and a threshold vector $\theta$ in such a way that the associated dynamical system leaves the patterns $\xi^{(i)}, 1 \leq i \leq m$ invariant. In this setting, the (ANN) model works like an associative memory of the Hopfield type [2].

The main problem with the $(\mathrm{ANN})$ is the construction of the synaptic matrix, and the usual way to get a candidate consists in taking the Hebbian learning rule

$$
w_{i j}:=n^{-1} \sum_{r=1}^{m} \xi_{i}^{(r)} \xi_{j}^{(r)}, \quad i \neq j,
$$

and $w_{i i} \equiv 0$.

Assuming the prototypes $\xi^{(r)}, 1 \leq r \leq m$ orthogonal, we have

$$
W \xi^{(r)} \equiv \xi^{(r)}, r=1, . ., m
$$

and therefore, for a zero threshold $(\mathrm{ANN})$ the patterns are correctly memorized. It is worth noting that the $(\mathrm{ANN})$ can have other fixed points, and that Hebb's law cannot guarantee exact memorization of the prototypes when they are not orthogonal.

In the remaining we assume the following model: the components of the prototypes are independent and identically distributed random variables such that

$$
P\left(\xi_{i}^{(r)}=-1\right)=P\left(\xi_{i}^{(r)}=+1\right)=1 / 2 \quad \forall 1 \leq r \leq m
$$


and

$$
\xi_{i}^{(r)} \text { independent of } \xi_{j}^{(l)} \text { when } r \neq l \text { or } i \neq j \text {. }
$$

Moreover we will assume that $\theta=0$ and that the synaptic matrix is given by

$$
w_{i j} \equiv \frac{\sqrt{m}}{n} G\left(\frac{1}{\sqrt{m}} \sum_{r=1}^{m} \xi_{i}^{(r)} \xi_{j}^{(r)}\right), i \neq j,
$$

$w_{i i} \equiv 0$, where $G(\cdot)$ is some function (see e.g. [3] or [4]). $G(x) \equiv x$ corresponds to the Hebbian learning rule and $G(x) \equiv \operatorname{Sgn}(x)$ corresponds to the so-called clipped Hebbian rule which is well known in practical applications (see [1] or $[5])$.

The capacity of such a random $(\mathrm{ANN})$ can be viewed under various angles ([5], [6], [7], [8]). Here we deal with direct attraction, and our aim is to find conditions on $m=m(n), m(n) \rightarrow \infty$ as $n \rightarrow \infty$, in such a way that

$$
P\left(\xi^{(1)}=\operatorname{Sgn}\left(W \xi^{(1)}\right)\right) \longrightarrow 1 \text { as } n \rightarrow \infty,
$$

where $\operatorname{Sgn}(\cdot)$ is taken componentwise, that is $\xi^{(1)}$ is a fixed point of the dynamical system. For the Hebbian rule, retrieval without errors occurs when

$$
m<\frac{n}{2 \log (n)}, n \rightarrow \infty, m \rightarrow \infty,
$$

(see [5]), and, for the clipped Hebbian rule, when [9]

$$
m<\frac{n}{\pi \log (n)}, n \rightarrow \infty, m \rightarrow \infty
$$

In the long term attraction context, Sompolinsky [4] considered (ANN) models with $m=\alpha n$, for some $\alpha \in \mathbb{N}$, and with synaptic weights $v_{i j}$ of the form

$$
v_{i j} \equiv \frac{\sqrt{m}}{n} G\left(\frac{1}{\sqrt{m}} \sum_{r=1}^{m} \xi_{i}^{(r)} \xi_{j}^{(r)}\right)+\eta_{i j},
$$

where the external contribution $\eta_{i j}$ is assumed to be independent of the learning algorithm, and is a random spin-glass like interaction. Using the replica mean field theory, Sompolisky obtained that at zero temperature the above random network is equivalent to a network with linear synapses and static Gaussian noise $\delta_{i j}$ independent of the memories, and observed that for large $m \in \mathbb{N}$ the most interesting source of noise is the nonlinearity of the learning algorithm. It's strength depends of the difference

$$
E\left(G(N)^{2}\right)-E(N G(N))^{2} \geq 0
$$

where $N$ denotes a standard normal random variable. In our context, we will use large deviations methods for estimating the probability (1.5). Our main result 
is that for bounded synaptic functions $G$ with bounded continuous derivative $G^{\prime}$ satisfying $E\left(G^{\prime}(N)\right)=E(N G(N))>0$, retrieval without errors occurs when

$$
m<\frac{n}{2 \log (n)} q_{G}, n \rightarrow \infty, m \rightarrow \infty,
$$

where

$$
q_{G}:=\frac{E(N G(N))^{2}}{E\left(G(N)^{2}\right)} \leq 1,
$$

which yields a lower bound for the capacity of the (ANN). Remark that the right side of (1.8) gives the true capacities (1.6) and (1.7).

\section{Preliminaries}

Let

$$
h_{i}(x):=\sum_{j \neq i} w_{i j} x_{j}
$$

be the post synaptic potential. Direct attraction takes place when $\xi_{i}^{(1)}=$ $\operatorname{Sgn}\left(h_{i}\left(\xi^{(1)}\right)\right.$, or equivalently when

$$
S_{i}^{(1)}:=\xi_{i}^{(1)} h_{i}\left(\xi^{(1)}\right)=\xi_{i}^{(1)} \sum_{j \neq i} \xi_{j}^{(1)} G\left(\frac{1}{\sqrt{m}} \sum_{r=1}^{m} \xi_{i}^{(r)} \xi_{j}^{(r)}\right)>0, i=1, . ., n .
$$

Then (1.5) becomes

$$
P\left(S_{1}^{(1)}>0, . ., S_{n}^{(1)}>0\right) \longrightarrow 1 \quad \text { when } n \rightarrow \infty .
$$

In our approach we will use the simple inequality

$$
1-n P\left(S_{1}^{(1)} \leq 0\right) \leq 1-P\left(\exists 1 \leq i \leq n, S_{i}^{(1)} \leq 0\right)=P\left(S_{1}^{(1)}>0, . ., S_{n}^{(1)}>0\right) \leq 1,
$$

and find conditions on $m=m(n)$ in such a way that

$$
n P\left(S_{1}^{(1)} \leq 0\right) \longrightarrow 0 \quad \text { as } \quad n \rightarrow \infty .
$$

Clearly we will only obtain a lower bound for the storage capacity, but it may be well that it corresponds to the exact capacity. A motivation for this is that under the assumption that the events $\left\{S_{1}^{(1)}<0\right\}, . .,\left\{S_{n}^{(1)}<0\right\}$ are asymptotically independent (as it is the case for the Hebbian learning rule [5]), we get that $P\left(S_{1}^{(1)}>0, . ., S_{n}^{(1)}>0\right) \sim\left(1-P_{1}\right)^{n}$ which converges to 1 when $n P_{1} \longrightarrow 0$.

Given $\left\{a^{r} \in\{-1,+1\}\right\}_{r=1, . ., m}$, consider the event

$$
A:=\left\{\xi_{1}^{(r)}=a^{r}, r=1, . ., m\right\} .
$$


On $A, S_{1}^{(1)}$ becomes

$$
\sum_{j=2}^{n} a^{1} \xi_{j}^{(1)} G\left(\frac{1}{\sqrt{m}} \sum_{r=1}^{m} a^{r} \xi_{j}^{(r)}\right)
$$

and the random variables

$$
z_{A, j}:=a^{1} \xi_{j}^{(1)} G\left(\frac{1}{\sqrt{m}} \sum_{r=1}^{m} a^{r} \xi_{j}^{(r)}\right), 2 \leq j \leq n,
$$

are independent and identically distributed. Moreover by symmetry the distribution of these variables does not depend on $A$, and therefore $P_{1}$ becomes

$$
P_{1}:=P\left(S_{1}^{(1)} \leq 0\right)=P\left(\sum_{j=2}^{n} x_{j}^{1} G\left(\frac{1}{\sqrt{m}} \sum_{r=1}^{m} x_{j}^{r}\right) \leq 0\right),
$$

where the $x_{j}^{r}, 2 \leq j \leq n, 1 \leq r \leq m$ are distributed as in (1.2).

\section{Moderate Deviations}

Let

$$
y_{i, m}:=x_{i}^{1} G\left(\frac{1}{\sqrt{m}} \sum_{r=1}^{m} x_{i}^{r}\right), \quad i=2, . ., n,
$$

with

$$
\lambda_{m}:=E\left(y_{i, m}\right),
$$

and

$$
\tau_{m}^{2}:=\operatorname{Var}\left(y_{i, m}\right)
$$

We have

$$
\lambda_{m}=\frac{1}{2} E\left(G\left(\frac{1}{\sqrt{m}}+\frac{1}{\sqrt{m}} \sum_{r=2}^{m} x_{1}^{r}\right)\right)-\frac{1}{2} E\left(G\left(-\frac{1}{\sqrt{m}}+\frac{1}{\sqrt{m}} \sum_{r=2}^{m} x_{1}^{r}\right)\right) .
$$

Lemma 1 Let $G: \mathbb{R} \longrightarrow \mathbb{R}$ be continuous. Then $y_{i, m}$ converges weakly to $x G(N)$, where $x$ takes the values 1 and -1 with probability $1 / 2$ and $N$ is a standard normal random variable independent of $x$.

Proof: Let $F$ be bounded and continuous. Then

$$
E\left(F\left(y_{1, m}\right)\right)=E\left(F\left(G\left(\frac{1}{\sqrt{m}}+X_{m}\right)\right)\right)+E\left(F\left(-G\left(-\frac{1}{\sqrt{m}}+X_{m}\right)\right)\right),
$$

where $X_{m}:=(1 / \sqrt{m}) \sum_{r=2}^{m} x_{1}^{r}, m \in \mathbb{N}$. Using the weak convergence of $X_{m}$ to a standard normal random variable $N$, we get that

$$
E\left(F\left(y_{1, m}\right)\right) \longrightarrow E(F(G(N)))+E(F(-G(N)))=E(F(x G(N))),
$$

where $x$ takes the values -1 and +1 with probability $1 / 2$ and is independent of $N$. 
Assuming $G(\cdot)$ bounded and continuous, and using the weak convergence of $(1 / \sqrt{m})\left(1+\sum_{r=2}^{m} x^{r}\right)$ and $(1 / \sqrt{m})\left(-1+\sum_{r=2}^{m} x^{r}\right)$ to standard normal distributions $N(0,1)$, we get that

$$
\lambda_{m} \longrightarrow 0 \text { as } m \rightarrow \infty
$$

and

$$
\tau_{m}^{2} \longrightarrow \tau_{\infty}^{2}>0
$$

when $G(\cdot)$ is not trivial.

It is not hard to see that for the Hebbian rule $\lambda_{m}=(1 / \sqrt{m})$ and $\tau_{m}^{2}=$ $(m-1) / m$. Concerning the clipped Hebbian rule, assuming $m$ odd, we have that $\lambda_{m}=P\left(\sum_{r=2}^{m} x^{r}=0\right) \sim(1 / \sqrt{\pi m})$ and $\tau_{m}^{2}=1-\lambda_{m}^{2}$ (see e.g. [11]). In both cases

$$
\lambda_{m} \sim \operatorname{const} \frac{1}{\sqrt{m}},
$$

and we will see that this behavior still holds in the nonlinear case when $0<$ $E(N G(N))<\infty$, where the $N$ denotes a standard normal random variable.

We have

$$
P_{1}=P\left(S_{n, m}^{*}<-\frac{\sqrt{n-1} \lambda_{m}}{\tau_{m}}\right),
$$

where $S_{n, m}^{*}$ denotes the normalized sum

$$
S_{n, m}^{*}:=\sum_{i=2}^{n} \frac{\left(y_{i, m}-\lambda_{m}\right)}{\sqrt{n-1} \tau_{m}} .
$$

Set

$$
x(n, m):=\frac{\sqrt{n-1} \lambda_{m}}{\tau_{m}} .
$$

Theorem 1 Assume that $G$ is bounded and continuous, and let $m=m(n)$ be such that $m(n) \rightarrow \infty$ as $n \rightarrow+\infty$. Let $\beta(n), n \in \mathbb{N}$ be a sequence of real numbers with $\beta(n) \rightarrow+\infty$ as $n \rightarrow \infty$ and $\beta(n)=o\left(n^{1 / 6}\right)$. Then

$$
P\left(S_{n, m}^{*}<-\beta(n)\right) \sim P(N<-\beta(n)),
$$

where $N$ denotes a standard normal random variable.

The proof is refered to the Appendix.

Following Hebb [10], correlated neuronal activities increase the synaptic weights, which means that $w_{i j}$ should increase whenever neuron $i$ and $j$ have the same activity, and decrease otherwise, what amounts to saying that $G(\cdot)$ should be increasing. In our context, we will translate Hebb's principle by saying $\lambda_{m}$ asymptotically positive (as it will become clearer in the sequel), that is there exists an integer $m_{0}$ such that

$$
\lambda_{m}>0 \quad \forall m>m_{0} .
$$


In view of (3.4) we see for example that $\lambda_{m}$ is asymptotically positive when $G$ is increasing. Then $x(n, m)$ is asymptotically positive and, when $x(n, m)=$ $o\left(n^{1 / 6}\right)$, we can envisage the approximation

$$
P_{1} \sim Q(x(n, m(n))),
$$

where

$$
Q(x) \equiv \frac{1}{\sqrt{2 \pi}} \int_{x}^{\infty} e^{-\frac{x^{2}}{2}} d x,
$$

and using the approximation [11]

$$
Q(x) \sim \frac{1}{\sqrt{2 \pi}} \frac{1}{x} e^{-\frac{x^{2}}{2}}, \quad x \rightarrow+\infty,
$$

we get that

$$
P_{1} \sim \frac{1}{\sqrt{2 \pi}} \frac{1}{x(n, m(n))} e^{-\frac{x(n, m(n))^{2}}{2}} .
$$

Theorem 2 Assume that $G$ is bounded with bounded continuous derivative $G^{\prime}$ satisfying $E\left(G^{\prime}(N)\right)=E(N G(N))>0$. Then direct attraction occurs (see 1.5) when $m=m(n) \longrightarrow \infty$ in such a way that

$$
m \leq \frac{n}{2 \log (n)} q_{G}, \quad n \rightarrow \infty
$$

with

$$
0<q_{G}:=\frac{(E(N G(N)))^{2}}{E\left(G^{2}(N)\right)} \leq 1,
$$

(recall that $(n /(2 \log (n)))$ is the capacity associated with the Hebbian learning rule).

Proof: Let $X_{m}:=(1 / \sqrt{m}) \sum_{r=2}^{m} x^{r}, m \in \mathbb{N}$. By the mean value Theorem there exists $\alpha_{m}\left(X_{m}\right) \in\left(X_{m}-(1 / \sqrt{m}), X_{m}+(1 / \sqrt{m})\right)$ such that

$$
G\left(X_{m}+(1 / \sqrt{m})\right)-G\left(X_{m}-(1 / \sqrt{m})\right)=\frac{2}{\sqrt{m}} G^{\prime}\left(\alpha_{m}\left(X_{m}\right)\right)
$$

The above expression implies that the function $G^{\prime}\left(\alpha_{m}\left(X_{m}\right)\right)$ is measurable. By construction $X_{m} \in\{(i / \sqrt{m}) ; i \in Z,-m \leq i \leq m\}$, and takes only a finite number of values. $\alpha_{m}\left(X_{m}\right)$ takes then only a finite number of values and is therefore measurable. By construction $X_{m}-(1 / \sqrt{m})<\alpha_{m}\left(X_{m}\right)<$ $X_{m}+(1 / \sqrt{m})$, and both $X_{m}-(1 / \sqrt{m})$ and $X_{m}+(1 / \sqrt{m})$ weakly converge to standard normal distributions. Thus $\alpha_{m}\left(X_{m}\right)$ converges weakly to a standard normal random variable $N$ and therefore

$$
E\left(G^{\prime}\left(\alpha_{m}\left(X_{m}\right)\right)\right) \longrightarrow E\left(G^{\prime}(N)\right)=E(N G(N))
$$


since by assumption $G^{\prime}$ is bounded continuous, and it follows that

$$
\lambda_{m} \sim E(N G(N)) / \sqrt{m} .
$$

The sequence $\left(\lambda_{m}\right)_{m \in \mathbb{N}}$ is thus asymptotically positive, and by (3.10)

$$
P_{1} \sim \frac{1}{\sqrt{2 \pi}} \frac{\tau_{\infty} \sqrt{m}}{\sqrt{n-1} E(N G(N))} \exp \left(-\frac{(n-1) E(N G(N))^{2}}{2 m \tau_{\infty}}\right),
$$

which shows that $n P_{1} \longrightarrow 0$ whenever

$$
m \leq \frac{n}{2 \log (n)} q_{G},
$$

with $q_{G} \leq 1$ by Cauchy-Schwarz.

Lemma 2 Let $G: \mathbb{R} \longrightarrow \mathbb{R}$ be such that $|E(N G(N))|<\infty$ and $E\left(G(N)^{2}\right)<$ $\infty$, and let

$$
G_{\circ}(x) \equiv \frac{1}{2}(G(x)-G(-x)) .
$$

Then

$$
q_{G} \leq q_{G_{0}} \leq 1 .
$$

Proof: Let $G_{e}(x):=(1 / 2)(G(x)+G(-x))$ be the even part of $G$, and $G_{0}$ be its odd part. We have

$$
\begin{aligned}
E(N G(N)) & =E\left(N\left(G_{0}+G_{e}\right)(N)\right) \\
& =E\left(N G_{0}(N)\right)+E\left(N G_{e}(N)\right) \\
& =E\left(N G_{0}(N)\right),
\end{aligned}
$$

since the function $x G_{e}(x)$ is odd. Moreover

$$
\begin{aligned}
E\left(G(N)^{2}\right) & =E\left(G_{o}(N)^{2}+G_{e}(N)^{2}\right)+E\left(G_{o}(N) G_{e}(N)\right) \\
& =E\left(G_{0}(N)^{2}+G_{e}(N)^{2}\right) \\
& \geq E\left(G_{o}(N)^{2}\right)
\end{aligned}
$$

since the function $G_{o}(x) G_{e}(x)$ is odd.

Theorem 3 Assume that $G$ is bounded and continuous, and that the sequence $\lambda_{m}, m \in I N$, is asymptotically non-positive. Let $m=m(n)$ be such that $m(n) \longrightarrow \infty$ as $n \rightarrow \infty$. Then, $\forall 0<\delta<\frac{1}{2}$ there exists $n_{0} \in \mathbb{N}$ such that

$$
P\left(\xi^{(1)}=\operatorname{Sgn}\left(W \xi^{(1)}\right)\right)<\frac{1}{2}+\delta, \forall n \geq n_{0} .
$$


Proof: Let $S_{n, m(n)}:=\sum_{i=2}^{n} z_{i, m(n)}$, where $z_{i, m(n)}:=y_{i, m(n)}-\lambda_{m}$, with $E\left(z_{i, m(n)}\right)=0$ and $\operatorname{Var}\left(z_{i, m(n)}\right)=\tau_{m}^{2} \longrightarrow \tau_{\infty}^{2}>0$ when $G$ is not trivial. Let $s_{n}^{2}=(n-1) \tau_{m}^{2}$ be the variance of the sum $S_{n, m(n)}$. Lindeberg's theorem (see e.g. [12]) asserts that $S_{n, m(n)} / s_{n}=S_{n, m(n)}^{*}$ weakly converges to a standard normal random variable if

$$
\frac{1}{s_{n}^{2}} \sum_{i=2}^{n} \int_{\left\{\left|z_{i, m(n)}\right| \geq \varepsilon s_{n}\right\}} z_{i, m(n)}^{2} d P \longrightarrow 0,
$$

as $n \rightarrow \infty, \forall \varepsilon>0$. Using the fact that $G$ is bounded we get that there exists a positive constant $C \in \mathbb{R}$ such that $\left|z_{i, m(n)}\right|<C, \forall 2 \leq i \leq n, \forall n \in \mathbb{N}$. It follows that

$$
\frac{1}{s_{n}^{2}} \sum_{i=2}^{n} \int_{\left\{\left|z_{i, m(n)}\right| \geq \varepsilon s_{n}\right\}} z_{i, m(n)}^{2} d P<\frac{C^{2}}{\tau_{m(n)}^{2}} P\left(\left|z_{1, m(n)}\right| \geq \varepsilon s_{n}\right) .
$$

But $P\left(\left|z_{1, m(n)}\right| \geq \varepsilon s_{n}\right)=0$ for $n \in \mathbb{N}$ satisfying $C<s_{n} \varepsilon$, that is for $n \geq n_{1}$ for some $n_{1} \in \mathbb{N}$, which proves (3.11). By assumption there exists $n_{2} \in \mathbb{I N}$ such that $x(n, m) \leq 0, \forall n \geq n_{2}$, and therefore

$$
P_{1}=P\left(S_{n, m(n)}^{*} \leq-x(n, m(n))\right) \geq P\left(S_{n, m(n)}^{*} \leq 0\right) \longrightarrow \frac{1}{2}
$$

and therefore

$$
\begin{aligned}
P\left(S_{1}^{(1)}>0, . ., S_{n}^{(1)}>0\right) & \leq P\left(S_{1}^{(1)}>0\right)=1-P\left(S_{1}^{(1)} \leq 0\right) \\
& \leq 1-P\left(S_{n, m(n)}^{*} \leq 0\right)-\frac{1}{2}
\end{aligned}
$$

which shows the result.

Example 1 Assume that $G$ is even. Then $E(N G(N))=0$ and $\lambda_{m}=0$, $\forall m \in I N$.

Example 2 Let $G$ be bounded with bounded continuous derivative $G^{\prime}$ satisfying $E\left(G^{\prime}(N)\right)=E(N G(N))<0$. Then the sequence $\lambda_{m}, m \in I N$ is asymptotically negative (for the proof, proceed as in proof of Theorem 2). Take for example $G$ to be decreasing.

Remark 1 As we have seen, $\lambda_{m}$ is asymptotically positive when $E(N G(N))>$ 0 and asymptotically negative when $E(N G(N))<0$. We suspect that both situations can happen in the critical case $E(N G(N))=0$. 


\section{Appendix}

\section{Proof of Theorem 1}

We proceed mainly as in Feller, volume II, chapter 7 , Theorem 1 [11]. Let $z_{m}:=y_{m}-\lambda_{m}$, where $y_{m}:=x^{1} G\left((1 / \sqrt{m}) \sum_{r=1}^{m} x^{r}\right), m \in \mathbb{N}$, and $\lambda_{m}:=$ $E\left(y_{m}\right) \longrightarrow 0$ as $m \rightarrow \infty$ since by assumption $G$ is bounded and continuous. For the same reason, $\tau_{m} \longrightarrow \tau_{\infty}>0$, when $G$ is not trivial. Let $F_{m}(d x)$ be the distribution associated with $z_{m}, m \in \mathbb{N}$, and let $F_{n, m}(d x)$ be that of the normalized sum $S_{n, m}^{*}$. Since for each $m \in \mathbb{N}$ the variable $z_{m}$ takes only a finite number of values, the moment generating function

$$
f_{m}(s) \equiv E\left(\exp \left(s z_{m}\right)\right),
$$

exists $\forall s \in \mathbb{R}$ and is analytic on $\mathbb{R}$ (finite sum of exponentials). The same property also holds for the logarithmic moment generating function

$$
\psi_{m}(s) \equiv \log \left(f_{m}(s)\right) .
$$

We have

$$
\psi_{m}^{(1)}(s) \equiv \frac{f_{m}^{(1)}(s)}{f_{m}(s)}
$$

where $\psi_{m}^{(k)}, k \in \mathbb{N}$, denotes the $k$ th derivative of $\psi$, and therefore

$$
\psi_{m}^{(1)}(0)=f_{m}^{(1)}(0)=0,
$$

since by construction $E\left(z_{m}\right)=0 \forall m \in \mathbb{N}$. In the same way we get that

$$
\psi_{m}^{(2)}=E\left(z_{m}^{2}\right)=\tau_{m}^{2}>0
$$

The Taylor expansion of $\psi_{m}$ around the origin takes then the form

$$
\psi_{m}(s) \equiv \frac{1}{2} \tau_{m}^{2} s^{2}+\frac{\psi_{m}^{(3)}(0)}{3 !} s^{3}+. .
$$

As in Feller [11] consider the associated random variable $w_{m}^{s}, m \in \mathbb{N}, s \in \mathbb{R}$, with distribution $V_{m}^{s}(d x)$ given by

$$
V_{m}^{s}(d x):=\frac{\exp (s x)}{f_{m}(s)} F_{m}(d x), x \in \mathbb{R}, s<0,
$$

and moment generating function

$$
v_{m}^{s}(\xi) \equiv \frac{f_{m}(\xi+s)}{f_{m}(s)} .
$$


The $w_{m}^{s}$ has expectation $\psi_{m}^{(1)}(s)$ and variance $\psi_{m}^{(2)}(s)$. Let us turn to sums of such random variables. Let $F_{m}^{n}(d x)$ be the distribution of the sum $z_{1, m}+. .+$ $z_{n, m}$, where the $z_{i, m}$ are i.i.d with distribution $F_{m}(d x)$, and let $F_{m}^{s, n}(d x)$ be the distribution of $w_{1, m}^{s}+. .+w_{n, m}^{s}$, where the $w_{i, m}^{s}$ are i.i.d. distributed according to $V_{m}^{s}(d x)$. By construction

$$
F_{n, m}(x)=F_{m}^{n}\left(\tau_{m} \sqrt{n} x\right), x \in \mathbb{R} .
$$

Let us check that

$$
F_{n, m}(x)=e^{n \psi_{m}(s)} \int_{-\infty}^{x \tau_{m} \sqrt{n}} e^{-s y} V_{m}^{s, n}(d y) .
$$

Let $f_{m}^{n}$ be the moment generating function associated with the sum $z_{1, m}+\ldots+$ $z_{n, m}$, and let $f_{m}^{s, n}$ be that of $w_{1, m}^{s}+. .+w_{n, m}^{s}$. Then $f_{m}^{n}(\xi+s) \equiv\left(f_{m}(\xi+s)\right)^{n}$, and therefore

$$
\frac{f_{m}^{n}(\xi+s)}{f_{m}^{n}(s)} \equiv\left(\frac{f_{m}(\xi+s)}{f_{m}(s)}\right)^{n} \equiv v_{m}^{s}(\xi)^{n},
$$

$s \in \mathbb{R}$ (see 4.5). It follows that $f_{m}^{s, n}$ (the moment generating function associated with $V_{m}^{s, n}$ ) is the same as that of the distribution given by

$$
\frac{\exp (s x) F_{m}^{n}(d x)}{E\left(\exp \left(s\left(z_{1, m}+. .+z_{n, m}\right)\right)\right)}=\frac{\exp (s x)}{\exp \left(n \psi_{m}(s)\right)} F_{m}^{n}(d x)
$$

and therefore

$$
V_{m}^{s, n}(d x)=\frac{\exp (s x)}{\exp \left(n \psi_{m}(s)\right)} F_{m}^{n}(d x)
$$

which yields (4.6).

Following Feller, we will replace $V_{m}^{s, n}(d x)$ by a normal distribution with expectation $n \psi_{m}^{(1)}(s)$ and variance $n \psi_{m}^{(2)}(s)$, the relative error commited being small when the upper limit of the integral is close the the expectation $n \psi_{m}^{(1)}(s)$. In our context $x=-\beta<0$ and $\beta \rightarrow \infty$ as $n \rightarrow \infty$. Let $s \in \mathbb{R}$ and $x \in \mathbb{R}$ be linked by the relation

$$
\tau_{m} x=\psi_{m}^{(1)} \sqrt{n}, s<0, x<0 .
$$

By (4.3) $\psi_{m}$ is convex and decreases to 0 when $s \rightarrow 0$. This establishes a bijective relation between the variables $x<0$ and $s<0$ in a neighborhood $U_{m}(0)$ of the origin. To go a step further consider the Taylor expansion

$$
\psi_{m}^{(1)}(s)=\tau_{m}^{2} s+\int_{0}^{s}(s-t) \psi_{m}^{(3)}(t) d t
$$

The above expansion is justified for the folowing reason: by construction $\psi_{m}^{(1)}(s) \equiv$ $E\left(z_{m} \exp \left(s z_{m}\right)\right) / f_{m}(s)$, where $z_{m}$ takes only a finite number of values. Using 
the fact that $G$ is bounded we have $\left|z_{m}\right|=\left|x^{1} G\left((1 / \sqrt{m})\left(x^{1}+. .+x^{m}\right)\right)\right|<$ $C \forall m \in \mathbb{N}$, where $C$ is a positive constant. $\psi_{m}^{(1)}$ is thus analytic on $\mathbb{R}, \forall m \in \mathbb{N}$. Using the same argument we get that

$$
\sup _{s \in K, m \in \mathbb{N}}\left|\psi_{m}^{(i)}(s)\right|<M_{K}^{(i)}<+\infty, i=1,2,3,
$$

where $K$ denotes any compact set containing the origin. Thus (4.8) becomes

$$
\psi_{m}^{(1)}(s)=\tau_{m}^{2} s+O_{K}\left(s^{2}\right), s \in K,
$$

where $O_{K}$ denotes a term bounded by a constant depending only of $K$ times $s^{2}$. Thus (4.7) becomes

$$
\frac{\tau_{m} x}{\sqrt{n}}=\tau_{m}^{2} s+O_{K}\left(s^{2}\right), s \in K .
$$

For the same reason

$$
\psi_{m}^{(2)}(s)=\tau_{m}^{2}+O_{K}(|s|), s \in K
$$

and therefore $\psi_{m}^{(2)}(s)>0$ uniformly in $s \in K_{0} \subset K$ and $m \in \mathbb{N}$. This shows that for $s \in K_{0}$ relation (4.7) is one to one and we can write

$$
s \sim \frac{x}{\tau_{\infty} \sqrt{n}} \text { if } \frac{x}{\sqrt{n}} \longrightarrow 0, s \in K_{0} .
$$

Next let us replace in (4.6) the distribution $V_{m}^{s, n}(d x)$ by the normal distribution with expectation $n \psi_{m}^{(1)}(s)$ and variance $n \psi_{m}^{(2)}(s)$, that is consider the expression

$$
A_{m}^{s, n}:=\exp \left(n \psi_{m}(s)\right) \int_{-\infty}^{x \tau_{m} \sqrt{n}} e^{-s y} \frac{1}{\sqrt{2 \pi}} \exp \left(-\frac{\left(y-n \psi_{m}^{(1)}(s)\right)^{2}}{2 n \psi_{m}^{(2)}(s)}\right) d y .
$$

Using the change of variable $t=n \psi_{m}^{(1)}(s)+y \sqrt{n \psi_{m}^{(2)}(s)}$, we get that

$$
A_{m}^{s, n}=\exp \left(n\left(\psi_{m}(s)-s \psi_{m}^{(1)}(s)+\frac{1}{2} s^{2} \psi_{m}^{(2)}(s)\right)\right) \mathcal{N}\left(s \sqrt{n \psi_{m}^{(2)}(s)}\right)
$$

where $\mathcal{N}$ denotes the standard normal distribution. Using the expansion $\psi_{m}(s)=$ $(1 / 2) \tau_{m}^{2} s^{2}+O_{K}\left(|s|^{3}\right),(4.10)$ and (4.11) we get that, for $s \in K_{0}$,

$$
n\left(\psi_{m}(s)-s \psi_{m}^{(1)}(s)+\frac{1}{2} s^{2} \psi_{m}^{(2)}(s)\right)=O_{K}\left(n|s|^{3}\right),
$$

which yields the estimate

$$
A_{m}^{s, n}=\mathcal{N}\left(s \sqrt{n \psi_{m}^{(2)}(s)}\right)\left(1+O_{K}\left(|s|^{3}\right), s \in K_{0},\right.
$$


$s<0, s \rightarrow 0, n \rightarrow \infty, m \rightarrow \infty$. When $n s^{3} \rightarrow 0$, what amounts to saying (see $(4.12))$

$$
x=o\left(n^{\frac{1}{6}}\right)
$$

we can write

$$
A_{m}^{s, n}=\mathcal{N}(\bar{x})\left(1+O_{K}\left(\frac{|x|^{3}}{\sqrt{n}}\right)\right), s \in K_{0}
$$

where

$$
\bar{x}:=s \sqrt{n \psi_{m}^{(2)}(s)}, s \in K_{0} .
$$

Following Feller, let us estimate the error commited by replacing $\bar{x}$ by $x$ in the above expression. We have

$$
\begin{aligned}
|\bar{x}-x| & =\left|s \sqrt{n \psi_{m}^{(2)}(s)}-\frac{\psi_{m}^{(1)}(s) \sqrt{n}}{\tau_{m}}\right| \\
& =\sqrt{n}\left|s \sqrt{\tau_{m}^{2}+O_{K}(|s|)}-t a u_{m} s+O_{K}\left(s^{2}\right)\right| \\
& =\sqrt{n}|s|\left|\sqrt{\tau_{m}^{2}+O_{K}(|s|)}-\tau_{m}+O_{K}(|s|)\right| \\
& =\sqrt{n}|s| O_{K}(|s|)=\sqrt{n} O_{K}\left(s^{2}\right) \\
& =O_{K}\left(\frac{x^{2}}{\sqrt{n}}\right)
\end{aligned}
$$

(see (4.10), (4.11) and (4.12)). It remains to consider the ratio $\mathcal{N}(\bar{x}) / \mathcal{N}(x)$. We have

$$
|\log | \frac{\mathcal{N}(\bar{x})}{\mathcal{N}(x)}||=O(|x||\bar{x}-x|)=O\left(\frac{|x|^{3}}{n}\right),
$$

which yields the estimate $|\mathcal{N}(\bar{x}) / \mathcal{N}(x)|=1+O\left(|x|^{3} / n\right)$. It follows that (see (4.16))

$$
A_{m}^{s, n}=\mathcal{N}(x)\left(1+O_{K}\left(\frac{|x|^{3}}{\sqrt{n}}\right),\right.
$$

when $n \rightarrow \infty, m \rightarrow \infty, s \in K_{0}, s<0, s \rightarrow 0$ in such a way that $x=o\left(n^{1 / 6}\right)$ (see (4.15)).

Returning to (4.6) we must now estimate the integral

$$
J:=\exp \left(n \psi_{m}(s)\right) \int_{-\infty}^{x \tau_{m} \sqrt{n}} e^{-s y}\left(V_{m}^{s, n}(d y)-\mathcal{N}_{m}^{s, n}(d y)\right)
$$

where $\mathcal{N}_{m}^{s, n}(d y)$ is the distribution of the normal random variable with expectation $n \psi_{m}^{(1)}(s)$ and variance $n \psi_{m}^{(2)}(s)$. By the Berry-Essen Theorem

$$
\left|V_{m}^{s, n}(t)-\mathcal{N}_{m}^{s, n}(t)\right|<\frac{3 M_{m}^{s}}{\sqrt{n} \tau_{m}^{3}}, \forall m \in \mathbb{N}, \forall t \in \mathbb{R},
$$


where $M_{m}^{s}$ denotes the third absolute moment of $V_{m}^{s}$. Let $w_{m}^{s}$ be distributed according to $V_{m}^{s}$. Then we have

$$
M_{m}^{s}=\int_{\mathbb{R}}|w|^{3} \frac{\exp (s w)}{E\left(\exp \left(s z_{m}\right)\right)} F_{m}(d w)
$$

But $\operatorname{supp}\left(F_{m}\right) \subset\{G(x),-G(x) ; x \in \mathbb{R}\}$ and it follows that

$$
\sup _{m \in \mathbb{N}}\left\{|w|^{3} ; F_{m}(w)>0\right\}<+\infty,
$$

since by assumption $G$ is bounded. Thus

$$
\sup _{s \in K_{0}, m \in \mathbb{N}} M_{m}^{s}<+\infty,
$$

which yields the uniform bound

$$
\sup _{s \in K_{0}, m \in \mathbb{N}}\left|V_{m}^{s, n}(t)-\mathcal{N}_{m}^{s, n}(t)\right|<\frac{D}{\sqrt{n}}, \forall t \in \mathbb{R},
$$

where $D$ is a positive constant. For the normal distribution we have

$$
\begin{aligned}
\int_{-\infty}^{n \psi_{m}^{(1)}(s)} e^{-s y} \mathcal{N}_{m}^{s, n}(d y)= & \exp \left(-s n \psi_{m}^{(1)}(s)\right) \mathcal{N}_{m}^{s, n}\left(n \psi_{m}^{(1)}(s)\right) \\
& +s \int_{-\infty}^{n \psi_{m}^{(1)}(s)} e^{-s y} \mathcal{N}_{m}^{s, n}(y) d y
\end{aligned}
$$

Let $\Lambda:=\operatorname{supp}\left(V_{m}^{s, n}\right) \cap\left(-\infty, n \psi_{m}^{(1)}(s)\right]$. When $\Lambda \neq \emptyset$ set $\Lambda=\left\{y_{1}<y_{2}<. .<\right.$ $\left.y_{p}\right\}, y_{p} \leq n \psi_{m}^{(1)}(s)$. If $\Lambda=\emptyset$ the integral

$$
\int_{-\infty}^{n \psi_{m}^{(1)}(s)} e^{-s y} V_{m}^{s, n}(d y)
$$

vanishes and, in the contrary, when $\Lambda \neq \emptyset$ we have

$$
I:=\int_{-\infty}^{n \psi_{m}^{(1)}(s)} e^{-s y} V_{m}^{s, n}(d y)=\sum_{i=1}^{p} e^{-s y_{i}} P\left(w_{m}^{s, n}=y_{i}\right) .
$$

When $\Lambda \neq \emptyset, V_{m}^{s, n}\left(n \psi_{m}^{(1)}(s)\right)=\sum_{i=1}^{p} P\left(w_{m}^{s, n}=y_{i}\right)$, and, more generally, $V_{m}^{s, n}(y)=\sum_{i=1}^{p(y)} P\left(w_{m}^{s, n}=y_{i}\right)$, where $p(y):=\sup \left\{k ; y_{k} \leq y\right\}$. Thus

$$
\begin{aligned}
I & =-\sum_{i=1}^{p-1} V_{m}^{s, n}\left(y_{i}\right)\left(e^{-s y_{i+1}}-e^{-s y_{i}}\right)+e^{-s y_{p}} V_{m}^{s, n}\left(y_{p}\right) \\
& =-\sum_{i=1}^{p-1} V_{m}^{s, n}\left(y_{i}\right)(-s) \int_{y_{i}}^{y_{i+1}} e^{-s y} d y+e^{-s y_{p}} V_{m}^{s, n}\left(y_{p}\right)+e^{-s y_{p}} V_{m}^{s, n}\left(y_{p}\right) \\
& =s \int_{-\infty}^{y_{p}} V_{m}^{s, n}(y) e^{-s y} d y+e^{-s y_{p}} V_{m}^{s, n}\left(y_{p}\right) .
\end{aligned}
$$


Returning to (4.18) we get

$$
\begin{aligned}
J \exp \left(-n \psi_{m}(s)\right)= & s \int_{-\infty}^{n \psi_{m}^{(1)}(s)} e^{-s y}\left(V_{m}^{s, n}(y)-\mathcal{N}_{m}^{s, n}(y)\right) d y \\
& +e^{-s n \psi_{m}^{(1)}(s)}\left(V_{m}^{s, n}\left(y_{p}\right)-\mathcal{N}_{m}^{s, n}\left(n \psi_{m}^{(1)}(s)\right)\right) \\
= & s \int_{-\infty}^{n \psi_{m}^{(1)}(s)} e^{-s y}\left(V_{m}^{s, n}(y)-\mathcal{N}_{m}^{s, n}(y)\right) d y \\
& +e^{-s n \psi_{m}^{(1)}(s)}\left(V_{m}^{s, n}\left(n \psi_{m}^{(1)}(s)\right)-\mathcal{N}_{m}^{s, n}\left(n \psi_{m}^{(1)}(s)\right)\right),
\end{aligned}
$$

and, using (4.20) we get that

$$
|J| \leq \frac{2 D}{\sqrt{n}} \exp \left(n\left(\psi_{m}(s)-s \psi_{m}^{(1)}(s)\right)\right)
$$

It follows that (see (4.14))

$$
\begin{aligned}
A_{m}^{s, n} & =\exp \left(n\left(\psi_{m}(s)-s \psi_{m}^{(1)}(s)\right)\right) \exp \left(\frac{\bar{x}^{2}}{2}\right) \mathcal{N}(\bar{x}) \\
& \sim \exp \left(n\left(\psi_{m}(s)-s \psi_{m}^{(1)}(s)\right)\right) \bar{x}^{-1}
\end{aligned}
$$

when $x=o\left(n^{(1 / 6)}\right)$, and thus the right side of (4.22) is $A_{m}^{s, n} O(|x| / \sqrt{n})$. Using (4.22) we get that

$$
\left|F_{n, m}(x)-A_{m}^{s, n}\right| \leq A_{m}^{s, n} O\left(\frac{|x|}{\sqrt{n}}\right),
$$

and therefore

$$
F_{n, m}(x)=A_{m}^{s, n}\left(1+O\left(\frac{|x|}{\sqrt{n}}\right)\right) .
$$

Finally, using (4.16) we get that

$$
\begin{aligned}
F_{n, m}(x) & =\mathcal{N}(\bar{x})\left(1+O\left(\frac{|x|^{3}}{\sqrt{n}}\right)\right)\left(1+O\left(\frac{|x|}{\sqrt{n}}\right)\right) \\
& =\mathcal{N}(x)\left(1+O\left(\frac{|x|^{3}}{\sqrt{n}}\right)\right),
\end{aligned}
$$

when $x=o\left(n^{1 / 6}\right)$ as required.

\section{References}

[1] M. Hasler. Recursive Neural Networks for Associative Memory. Wiley. 1990. 
[2] J. Hopfield. Neural Networks and physical systems with emergent collective computational abilities. Proc. Ntl. Acad. Sci. USA, vol. 79: pp. 2554-2558, 1982.

[3] D.J. Amit. Modeling Brain Function. The world of attractor neural networks. Cambridge University Press. 1992.

[4] H. Sompolinsky. Neural networks with nonlinear synapses and a static noise. Physical Review A. vol. 34, number 3, 1986.

[5] R. McEliece, E. Posner, E. Rodemich and S. Venkatesh. The capacity of the Hopfield associative memory. IEEE Trans. on Information Theory, vol. IT-33:pp. 461-482, 1987.

[6] C. Newman. Memory capacity in neural network models: rigorous lower bounds. Neural Networks, vol. 1:pp. 223-238, 1988.

[7] D. Louiakanova. Capacité de mémoire dans le modèle de Hopfield. C.R.A.S, 1994.

[8] O. Francois. New Rigorous Bounds for the Hopfield Neural Network Model. Preprint. LMC-IMAG, BP 53, 38041 Grenoble. France.

[9] P. Piret. Analysis of a Modified Hebbian Rule. IEEE Trans. on Information Theory, vol. 36, no. 6, 1990.

[10] D. Hebb. The organization of behavior. J. Wiley 1949.

[11] W. Feller. An Introduction to Probability Theory and Its Applications, vol. I and II, Wiley, 1971.

[12] P. Billingsley. Convergence of Probability Measures. Wiley. 1968.

\section{Current address:}

Christian Mazza

Section de Mathématiques

Université de Genève

2-4 Rue du Lièvre, Case Postale 240

CH-1211 Genève, Switzerland

e-mail: mazza@sc2a.unige.ch 